Relations industrielles

Industrial Relations

\title{
Collective Bargaining in the Public Service, Toronto, Ontario, The Institute of Public Administration of Canada, 1973, 105 pp.
}

Jean Boivin

Volume 28, numéro 3, 1973

URI : https://id.erudit.org/iderudit/028429ar

DOI : https://doi.org/10.7202/028429ar

Aller au sommaire du numéro

Éditeur(s)

Département des relations industrielles de l'Université Laval

ISSN

0034-379X (imprimé)

1703-8138 (numérique)

Découvrir la revue

Citer ce compte rendu

Boivin, J. (1973). Compte rendu de [Collective Bargaining in the Public Service, Toronto, Ontario, The Institute of Public Administration of Canada, 1973, 105 pp.] Relations industrielles / Industrial Relations, 28(3), 665-668.

https://doi.org/10.7202/028429ar

Tous droits réservés (C) Département des relations industrielles de l'Université Laval, 1973
Ce document est protégé par la loi sur le droit d'auteur. L’utilisation des services d'Érudit (y compris la reproduction) est assujettie à sa politique d'utilisation que vous pouvez consulter en ligne.

https://apropos.erudit.org/fr/usagers/politique-dutilisation/ 
viendrait à s'appliquer à l'entreprise postérieurement à la conclusion de la convention d'entreprise, cette dernière devra adapter ses dispositions moins favorables à celles de la convention nouvellement signée ou étendue par arrêté ministériel » (p. 207). D'autres lecteurs enfin, porteront leur attention sur ces sections du livre où l'auteur discute de l'impact que l'intégration européenne pourrait avoir sur les structures et modes d'action des syndicats européens. Parallèlement, la section du livre que l'auteur consacre à la «société anonyme enropéenne 》 pourrait intéresser vivement le spécialiste en relations industrielles, qu'il oeuvre de ce côté-ci, ou de l'autre, de l'Atlantique.

Mais tout est à lire dans l'ouvrage de Dimitri Weiss. Tout en laissant à l'auteur la responsabilité de ses thèses, de ses jugements, et de ses orientations, il faut reconnaître que ce remarquable et dense ouvrage, écrit dans une langue concise et claire, et donc de lecture agréable et captivante, réussit - tout en accordant une attention prioritaire aux problèmes tels qu'ils se posent en France, ce qui est normal puisque l'auteur est français - à faire passer sur le thème des «relations industrielles» le souffle serein d'une perspective internationale qui se veut avant tout fonctionnelle. Le livre de Dimitri Weiss mérite de recevoir bon accueil au Canada: dans nos Universités et nos institutions d'enseignement où il constituera un outil de travail et de référence précieux; dans notre monde des 《relations industrielles », où il sera une source d'évaluations et d'informations bien intégrées et parfaitement actuelles.

Félix QUINET

Commission des relations de travail dans la fonction publique Ottawa

Le monde ouvrier au Québec, bibliographie rétrospective par André $\mathrm{E}$. LeBlanc et James D. Thwaites, Montréal, Les Presses de l'université du Québec, 1973, 283 pp.

Il y a quelques années la direction de Relations industrielles recevait pour publication une bibliographie sur les travailleurs au Québec. Grandement intéressé par le sujet, nous avons communiqué à l'auteur, André LeBlanc, nos commentaires avec des suggestions. Celui-ci a repris son texte et nous a présenté une nouvelle version grandement enrichie. C'est alors que nous avons mis l'auteur en contact avec un autre chercheur du département d'histoire de l'université Laval, James D. Thwaites, qui travaillait sur le même sujet. Le résultat de leur collaboration a pris une ampleur telle qu'il dépassait les cadres d'un article pouvant être publié dans Relations industrielles et fait aujourd'hui l'objet de l'ouvrage Le monde ouvrier au Québec bibliographie rétrospective édité par les Presses de l'université du Québec.

Après avoir suivi de près l'élaboration de cette recherche, nous ne pouvons que nous réjouir de sa publication. Ce n'est pas le premier ouvrage du genre. Sans être encore exhaustif, il nous apparaît le plus complet. Les auteurs ont relevé près de 2,500 titres qui ont été classés selon un ordre thématique comprenant huit divisions : le mouvement ouvrier; les syndicats et métiers; les conflits de travail; le travail; le milieu social; les réponses et réactions; la presse ouvrière ; bibliographies. Un excellent index analytique des auteurs et des groupements facilite la consultation.

Un ouvrage du genre comporte fatalement une bonne part d'arbitraire tant dans la délimitation du sujet que dans le choix des titres. En effet, il n est pas toujours facile de déterminer une frontière précise entre les études qui portent strictement sur le monde ouvrier au Québec et celles qui englobent tout le monde ouvrier au Canada dont celui du Québec. Il en va de même à propos de celles qui, tout en traitant un sujet plus vaste, apportent des éclairages importants sur le monde des travailleurs.

Une recherche du genre n'étant jamais terminée, il faut savoir gré aux auteurs de n'avoir pas attendu le chef d'oeuvre avant d'offrir aux chercheurs un instrument utile qui contribuera sûrement à faciliter leur travail.

\section{Université Laval}

Gérard DION

Collective Bargaining in the Public Service, Toronto, Ontario, The Institute of Public Administration of Canada, 1973, $105 \mathrm{pp}$.

Ce livre, dont la version française paraîtra prochainement, est le compte rendu d'un séminaire sur la négociation collective dans le secteur public qui avait 
tion publique du Canada du 16 au 18 juin 1972 à Québec. Le séminaire regroupait des experts en relations industrielles qui provenaient tant du milieu universitaire que des milieux syndicaux et patronaux.

Cinq conférences furent présentées et ce sont ces conférences qui sont publiées dans ce volume. Le premier article est celui de Thomas J. Plunkett, directeur de l'Institute of Local Government de l'Université Queen, et il porte sur la négociation collective dans les municipalités canadiennes. Il existe peu de littérature sur ce sujet au Canada et l'ouvrage du professeur Plunkett, même s'il n'est pas exhaustif, comble partiellement ce vide. On y décrit le cadre institutionnel dans lequel se déroule la négociation collective au niveau municipal de même que les principaux problèmes particuliers à ce secteur. Ainsi. après avoir dégagé les quatre principaux types d'unités de négociation qui existent à travers le pays, à savoir les employés manuels, les employés de bureau, les policiers et les pompiers, l'auteur fait l'inventaire des différents syndicats d'employés municipaux regroupant les travailleurs de ces unités de négociation.

Dans une autre partie de son article, le professeur Plunkett décrit les différents mécanismes de négociation et souligne que le problème le plus important dans ce secteur est celui du choix du porte parole patronal qui doit conduire les négociations avec les syndicats. Les facteurs qui affectent ce choix sont les suivants: le degré d'autorité que les conseils municipaux veulent bien déléguer à leurs administrateurs; la nature de la structure de gestion des municipalités; et les ressources administratives disponibles. Il va sans dire que les difficultés liées à la négociation collective croissent avec la densité des populations impliquées. Par ailleurs, la rigidité de la structure de revenus des municipalités, (ceux-ci dépendant surtout de la taxe foncière) impose une lourde contrainte aux négociateurs patronaux puisque les salaires et avantages sociaux des employés accaparent de $60 \%$ à $70 \%$ du budget municipal. Enfin, le comportement général des autorités municipales face à la négociation collective qui consiste à se contenter de répondre ou de réagir aux demandes de leurs employés plutôt que de chercher à établir un dia- logue permanent amène Plunkett à conclure que «As a rule, no machinery has evolved through which the unions or associations can carry on any kind of meaningful dialogue with municipal management relative to working conditions or other matters that have either not been included in the agreement or if they are included require some form of joint consultation as to their interpretation. Such discussions generally have to await the next round of negotiations with the result that relatively minor matters of importance to employees have a tendency to fester and develop subsequently into major issues 》 (p. 9).

Le second article de madame Shirley B. Goldenberg, professeur à la Faculty of Management de l'Université McGill, décrit les différents systèmes de négociations dans les services publics provinciaux. Il s'agit là d'un secteur où les modifications à la législation du travail ont été très nombreux depuis quelques années car déjà, depuis la rédaction de cet article, plusieurs provinces ont modifié les règles régissant la négociation collective entre les gouvernements provinciaux et leurs employés ou sont tout juste sur le point de le faire. Néanmoins, l'article de madame Goldenberg présente une valeur inestimable car c'est la première étude comparative des différents régimes de relations du travail qui couvre de façon exhaustive toutes les provinces canadiennes.

Dans la discussion des principaux problèmes communs aux différentes provinces, le professeur Goldenberg souligne notamment la question du conflit d'intérêt qui existe lorsque l'administration des procédures d'accréditation et de conciliation est laissée aux ministères du Travail. Sa suggestion à l'effet d'établir des commissions des relations du travail indépendantes du gouvernement a d'ailleurs déjà été retenue par la province du Nouveau Brunswick. Elle souligne également l'épineux problème du mandat des négociateurs gouvernementaux et suggère qu'il doit $\mathrm{y}$ avoir une délégation d'autorité à des négociateurs expérimentés qui soit suffisante pour inspirer confiance aux négociateurs syndicaux. Enfin, sur la question non moins épineuse du règlement des différends, madame Goldenberg mentionne que certaines provinces acceptent la procédure d'arbitrage obligatoire alors que d'autres, 
comme le Québec, la refusent. Elle mentionne à juste titre que retirer le droit de grève ne règlera pas le problème mais sa seule suggestion est à l'effet d'augmenter les pénalités dans les cas où la loi est violée ce qui, sur la base de l'expérience passée, est loin d'être une solution efficace.

Dans le troisième article, J.P. Connell du Conseil du trésor du Canada, décrit le régime de relations du travail en vigueur dans la fonction publique fédérale. Il s'agit d'une bonne source de documentation pour le profane mais on n'y retrouve à peu près aucune analyse. Le tout est présenté avec beaucoup de candeur et l'auteur semble prendre pour acquis que le régime de relations du travail de la fonction publique fédérale est le meilleur qui soit.

Le quatrième article porte sur la détermination des conditions de travail dans la fonction publique fédérale. Le directeur du Pay Research Bureau, T.J. Wilkins, y rapporte les résultats d'une recherche conduite par cet organisme sur les facteurs qui influencent la détermination des salaires et des avantages sociaux. Selon Wilkins, les facteurs les plus importants seraient les suivants : la législation (qui détermine le champ de la négociation); la politique salariale du gouvernement; la nécessité de maintenir une relation adéquate dans les conditions de travail des différents groupes occupationnels; l'influence de l'ancienne méthode (antérieure à la négociation collective) de revision des salaires à tous les deux ans; l'attitude de la population face à l'éventualité d'une grève ; et l'existence de deux organismes importants: le National Joint Council et le Pay Research Bureau. L'influence de ces facteurs est sans doute prépondérante mais on peut reprocher à l'auteur de ne pas avoir tenu compte d'autres facteurs tels l'impact du militantisme de certains groupes d'employés comme les postiers de même que le conservatisme paternaliste de certains autres comme les employés de bureau. Il semble que l'auteur ait voulu privilégier les facteurs objectifs au détriment des considérations liées au pouvoir de négociation des parties.

L'article contient également d'intéressantes observations et statistiques sur l'évolution des salaires et avantages sociaux depuis l'entrée en vigueur de la nouvelle législation en $196 \%$. Selon le directeur du Pay Research Bureau, il n'y aurait pas de différences importantes dans les augmentations de salaires obtenues par les syndicats qui ont choisi la conciliation par rapport à ceux qui ont choisi l'arbitrage obligatoire. De même, il n'y aurait pas plus de différences dans ce dernier cas entre les conventions signées au stade de la négociation avant l'arbitrage et les sentences arbitrales elles-mêmes.

Le cinquième article est signé de Marc Lapointe qui vient d'être nommé président du Conseil canadien des relations ouvrières. Cet article est rédigé dans un style très imagé et on $y$ découvre rapidement que l'auteur possède une vaste expérience des relations industrielles. Même si l'essentiel de son article porte sur une critique de l'arbitrage obligatoire et consiste en un plaidoyer en faveur du droit de grève dans son expression la plus traditionnelle, i.e. précédé d'une période de médiation ou de conciliation, les remarques les plus intéressantes se trouvent dans les considérations générales du début. En effet, Me Lapointe souligne quatre faits primordiaux que l'on a tendance à oublier lorsqu'on analyse les problèmes actuels de la négociation collective dans le secteur public.

D'abord, selon Me Lapointe, il faut se rappeler que les législateurs ont donné le droit d'association, parfois brusquement, à des masses énormes d'employés de la fonction publique, là où la syndicalisation était nulle ou larvée. Ce faisant, on a parfois coupé la base des dirigeants et 1 on n'a pas permis le développement normal et graduel d'un syndicalisme voulu et bien enraciné.

En second lieu, la plupart des législations, à quelques exceptions près, n'ont pas été précédées d'études appropriées qui auraient analysé le cadre social et politique dans lequel évolueraient les négociations collectives.

Ensuite, Me Lapointe affirme qu'on s'est précipité dans cette libéralisation de la négociation collective en partant de la prémisse générale que les mécanismes traditionnels étaient suffisamment adaptés ou adaptables pour conduire aux mêmes résultats que ceux obtenus dans l'entreprise privée. Le Québec, qui possède l'une des plus vieilles législations en matière de relations du travail pour les employés du secteur public, est en 
train de fournir la meilleure preuve de cette affirmation.

Enfin, et cette dernière remarque s'applique surtout au Québec, l'auteur avance que l'on a inscrit la négociation et les règles pour la diriger dans un contexte d'entreprise privée où la force d'impulsion dans les conditions de travail proviendrait et de la grande entreprise et d'un syndicalisme qui, parce que supposément 《d'affaires》, respecterait cette règle du jeu. Encore une fois cette affirmation est corroborée par la récente politisation du conflit mettant aux prises le gouvernement du Québec et le Front commun intersyndical.

Le dernier article qui sert également de conclusion à ce séminaire est celui de John Crispo, directeur du Centre de relations industrielles de l'Université de Toronto. Comme Me Lapointe, le professeur Crispo rejette le système d'arbitrage obligatoire institutionnalisé comme mécanisme de règlement des conflits d'intérêts dans le secteur public. Sa position est que l'on doit accorder (ou maintenir) le droit de grève, sauf dans les cas où les services essentiels sont menacés, car l'expérience canadienne révèle que notre société à date s'est fort bien accommodée des grèves dans le secteur public. Le professeur Crispo n'est pas très explicite quant aux procédures à suivre dans les cas où les services essentiels sont menacés, se contentant d'affirmer que la législation ne devrait rien prévoir de précis afin que les parties soient dans le doute quant au type d'intervention éventuelle. Cette solution nous semble trop facile, car elle escamotte le cas très important où le gouvernement provincial est en même temps l'employeur et celui qui doit décider de la forme d'intervention à utiliser.

La conclusion du professeur Crispo est très optimiste : en comparant la situation actuelle du secteur public avec celle du secteur privé il y a trente ans alors que le Canada et les Etats-Unis étaient affligés de nombreuses grèves, Celui-ci conclut qu'une période de stabilisation identique à celle qui a suivi ces années turbulentes dans le secteur privé, pourrait fort bien succéder au chaos actuel qui existe dans le secteur public.

\section{Université Laval}

Contemporary Collective Bargaining, by Harold W. Davey, New Jersey, Prentice-Hall Inc., Englewood Cliffs, 1972, $395 \mathrm{pp}$.

Ce livre constitue la troisième édition de cet ouvrage, la précédente datant déjà de 1959. Compte tenu du caractère dynamique de la négociation collective et de l'évolution constante du contenu des conventions qui en résultent, l'auteur n'a pas voulu se limiter à de simples retouches en vue d'atteindre à une mise-à-jour de son livre. En fait, l'édition qu'il nous présente maintenant constitue dans une large mesure une refonte complète de la précédente.

Sans avoir abandonné les thèmes traditionnels de la convention collective tels que les droits de la direction, le règlement des griefs, l'arbitrage et l'ancienneté, au sujet desquels il a parfois repris textuellement des extraits de certains chapitres de l'édition de 1959, l'auteur aborde dans le présent volume des sujets qui ont acquis ces dernières années une place importante dans la négociation. C'est ainsi par exemple qu'il accorde beaucoup plus d'importance qu'en 1959 aux problèmes relatifs à la sécurité d'emploi et aux bénéfices marginaux. Un chapitre entièrement nouveau est consacré à la négociation collective par les employés du gouvernement et par certaines catégories de professionnels tels que les enseignants et les infirmières.

En dépit de ces refontes et de ces additions, la perspective globale de l'ouvrage demeure sensiblement la même que dans l'édition précédente en ce sens que l'auteur paraît avoir tenté de maintenir le difficile juste milieu entre l'exposé purement théorique et l'analyse appliquée à des cas pratiques au risque même de décevoir quelque peu les uns et les autres, théoriciens et praticiens. Néanmoins les premiers y trouveront leur profit grâce aux abondantes références d'ordre bibliographique rapportées à la fin de chaque chapitre, et les seconds auront l'occasion de se familiariser avec une multitude de problèmes que pose la négociation collective au niveau de l'entreprise ou de l'établissement.

A cet égard, il s'agit d'un ouvrage dont il ne faut pas tant attendre une explication scientifique des phénomènes 